\title{
Sturm-Liouville BVP in Banach space
}

\author{
Hua Su', Lishan Liu ${ }^{2}$ and Xinjun Wang ${ }^{3}$
}

\footnotetext{
* Correspondence: jnsuhua@163. com

${ }^{1}$ School of Mathematic and Quantitative Economics, Shandong University of Finance and Economics, Jinan Shandong 250014, China

Full list of author information is available at the end of the article
}

\begin{abstract}
We consider the existence of single and multiple positive solutions for fourth-order Sturm-Liouville boundary value problem in Banach space. The sufficient condition for the existence of single and multiple positive solutions is obtained by fixed theorem of strict set contraction operator in the frame of the ODE technique. Our results significantly extend and improve many known results including singular and nonsingular cases.
\end{abstract}

\section{Introduction}

The boundary value problems (BVPs) for ordinary differential equations play a very important role in both theory and application. They are used to describe a large number of physical, biological, and chemical phenomena. In this article, we will study the existence of positive solutions for the following fourth-order nonlinear Sturm-Liouville BVP in a real Banach space $E$

$$
\left\{\begin{array}{l}
\frac{1}{p(t)}\left(p(t) u^{\prime \prime \prime}(t)\right)^{\prime}=f(u(t)), \quad 0<t<1 \\
\alpha_{1} u(0)-\beta_{1} u^{\prime}(0)=0, \gamma_{1} u(1)+\delta_{1} u^{\prime}(1)=0 \\
\alpha_{2} u^{\prime \prime}(0)-\beta_{2} \lim _{t \rightarrow 0+} p(t) u^{\prime \prime \prime}(t)=0 \\
\gamma_{2} u^{\prime \prime}(1)+\delta_{2} \lim _{t \rightarrow 1-} p(t) u^{\prime \prime \prime}(t)=0
\end{array}\right.
$$

where $\alpha_{i}, \beta_{i}, \delta_{i}, \gamma_{i} \geq 0(i=1,2)$ are constants such that $\rho_{1}=\beta_{1} \gamma_{1}+\alpha_{1} \gamma_{1}+\alpha_{1} \delta_{1}>0$, $B(t, s)=\int_{t}^{s} \frac{d \tau}{p(\tau)}, \rho_{2}=\beta_{2} \gamma_{2}+\alpha_{2} \gamma_{2} B(0,1)+\alpha_{2} \delta_{2}>0$, and $p \in C^{1}((0,1),(0,+\infty))$. Moreover $p$ may be singular at $t=0$ and/or 1 . BVP (1.1) is often referred to as the deformation of an elastic beam under a variety of boundary conditions, for detail, see [1-17]. For example, BVP (1.1) subject to Lidstone boundary value conditions $u(0)=u$ $(1)=u^{\prime \prime}(0)=u^{\prime \prime}(1)=0$ are used to model such phenomena as the deflection of elastic beam simply supported at the endpoints, see $[1,3,5,7-11,13,14]$. We notice that the above articles use the completely continuous operator and require $f$ satisfies some growth condition or assumptions of monotonicity which are essential for the technique used.

The aim of this article is to consider the existence of positive solutions for the more general Sturm-Liouville BVP by using the properties of strict set contraction operator. Here, we allow $p$ have singularity at $t=0,1$, as far as we know, there were fewer works to be done. This article attempts to fill part of this gap in the literature.

(C) 2011 Su et al; licensee Springer. This is an Open Access article distributed under the terms of the Creative Commons Attribution License (http://creativecommons.org/licenses/by/2.0), which permits unrestricted use, distribution, and reproduction in any medium, provided the original work is properly cited. 
This article is organized as follows. In Section 2, we first present some properties of the Green functions that are used to define a positive operator. Next we approximate the singular fourth-order BVP to singular second-order BVP by constructing an integral operator. In Section 3, the sufficient condition for the existence of single and multiple positive solutions for BVP (1.1) will be established. In Section 4, we give one example as the application.

\section{Preliminaries and lemmas}

In this article, we all suppose that $\left(E,\|\cdot\|_{1}\right)$ is a real Banach space. A nonempty closed convex subset $P$ in $E$ is said to be a cone if $\lambda P \in P$ for $\lambda \geq 0$ and $P \cap\{-P\}=\{\theta\}$, where $\theta$ denotes the zero element of $E$. The cone $P$ defines a partial ordering in $E$ by $x$ $\leq y$ iff $y-x \in P$. Recall the cone $P$ is said to be normal if there exists a positive constant $N$ such that $0 \leq x \leq y$ implies $\|x\|_{1} \leq N \mid\|y\|_{1}$. The cone $P$ is normal if every order interval $[x, y]=\{z \in E \mid x \leq z \leq y\}$ is bounded in norm.

In this article, we assume that $P \subseteq E$ is normal, and without loss of generality, we may assume that the normality of $P$ is 1 . Let $J=[0,1]$, and

$$
\begin{aligned}
& C(J, E)=\{u: J \rightarrow E \mid u(t) \text { continuous }\}, \\
& C^{i}(J, E)=\{u: J \rightarrow E \mid u(t) \text { is } i \text { - order continuously differentiable }\}, i=1,2, \ldots
\end{aligned}
$$

For $u=u(t) \in C(J, E)$, let $\|u\|=\max _{t \in J}\|u(t)\|_{1}$, then $C(J, E)$ is a Banach space with the norm $\|\cdot\|$.

Definition 2.1 A function $u(t)$ is said to be a positive solution of the BVP (1.1), if $u$ $\in C^{2}([0,1], E) \cap C^{3}((0,1), E)$ satisfies $u(t) \geq 0, t \in(0,1], p u^{\prime \prime \prime} \in C^{1}((0,1), E)$ and the $\operatorname{BVP}(1.1)$, i.e., $u \in C^{2}([0,1], P) \cap C^{3}((0,1), P)$ and $u(t) \not \equiv \theta, t \in J$.

We notice that if $u(t)$ is a positive solution of the BVP $(1.1)$ and $p \in C^{1}(0,1)$, then $u$ $\in C^{4}(0,1)$.

Now we denote that $H(t, s)$ and $G(t, s)$ are the Green functions for the following boundary value problem

$$
\left\{\begin{array}{l}
-u^{\prime \prime}=0, \quad 0<t<1, \\
\alpha_{1} u(0)-\beta_{1} u^{\prime}(0)=0, \quad \gamma_{1} u(1)+\delta_{1} u^{\prime}(1)=0
\end{array}\right.
$$

and

$$
\left\{\begin{array}{l}
\frac{1}{p(t)}\left(p(t) v^{\prime}(t)\right)^{\prime}=0, \quad 0<t<1 \\
\alpha_{2} v(0)-\lim _{t \rightarrow 0+} \beta_{2} p(t) v^{\prime}(t)=0 \\
\gamma_{2} v(1)+\lim _{t \rightarrow 1-} \delta_{2} p(t) v^{\prime}(t)=0
\end{array}\right.
$$

respectively. It is well known that $H(t, s)$ and $G(t, s)$ can be written by

$$
H(t, s)=\frac{1}{\rho_{1}}\left\{\begin{array}{l}
\left(\beta_{1}+\alpha_{1} s\right)\left(\delta_{1}+\gamma_{1}(1-t)\right), 0 \leq s \leq t \leq 1 \\
\left(\beta_{1}+\alpha_{1} t\right)\left(\delta_{1}+\gamma_{1}(1-s)\right), 0 \leq t \leq s \leq 1
\end{array}\right.
$$

and

$$
G(t, s)=\frac{1}{\rho_{2}}\left\{\begin{array}{l}
\left(\beta_{2}+\alpha_{2} B(0, s)\right)\left(\delta_{2}+\gamma_{2} B(t, 1)\right), 0 \leq s \leq t \leq 1 \\
\left(\beta_{2}+\alpha_{2} B(0, t)\right)\left(\delta_{2}+\gamma_{2} B(s, 1)\right), 0 \leq t \leq s \leq 1
\end{array}\right.
$$


where $\rho_{1}=\gamma_{1} \beta_{1}+\alpha_{1} \gamma_{1}+\alpha_{1} \delta_{1}>0, B(t, s)=\int_{t}^{s} \frac{\mathrm{d} \tau}{p(\tau)}, \rho_{2}=\alpha_{2} \delta_{2}+\alpha_{2} \gamma_{2} B(0,1)+\beta_{2} \gamma_{2}$ $>0$.

It is easy to verify the following properties of $H(t, s)$ and $G(t, s)$

(I) $G(t, s) \leq G(s, s)<+\infty, H(t, s) \leq H(s, s)<+\infty$;

(II) $G(t, s) \geq \rho G(s, s), H(t, s) \geq \xi H(s, s)$, for any $t \in[a, b] \subset(0,1), s \in[0,1]$, where

$$
\begin{aligned}
& \rho=\min \left\{\frac{\delta_{2}+\gamma_{2} B(b, 1)}{\delta_{2}+\gamma_{2} B(0,1)}, \frac{\beta_{2}+\alpha_{2} B(0, a)}{\beta_{2}+\alpha_{2} B(0,1)}\right\}, \\
& \xi=\min \left\{\frac{\delta_{1}+\gamma_{1}(1-b)}{\delta_{1}+\gamma_{1}}, \frac{\beta_{1}+\alpha_{1} a}{\beta_{1}+\alpha_{1}}\right\} .
\end{aligned}
$$

Throughout this article, we adopt the following assumptions $\left(H_{1}\right) p \in C^{1}((0,1),(0,+\infty))$ and satisfies

$$
0<\int_{0}^{1} \frac{\mathrm{d} s}{p(s)}<+\infty, 0<\lambda=\int_{0}^{1} G(s, s) p(s) \mathrm{d} s<+\infty
$$

$\left(H_{2}\right) f(u) \in C(P \backslash\{\theta\}, P)$ and there exists $M>0$ such that for any bounded set $B \subset C$ $(J, E)$, we have

$$
\alpha(f(B(t))) \leq M \alpha(B(t)), 2 M \lambda<1 .
$$

where $\alpha$ ( ) denote the Kuratowski measure of noncompactness in $C(J, E)$.

The following lemmas play an important role in this article.

Lemma 2.1 [17]. Let $B \subset C[J, E]$ be bounded and equicontinuous on $J$, then $\alpha(B)=\sup _{t \in J} \alpha(B(t))$.

Lemma 2.2 [16]. Let $B \subset C(J, E)$ be bounded and equicontinuous on $J$, let $\alpha(B)$ is continuous on $J$ and

$$
\alpha\left(\left\{\int_{J} u(t) \mathrm{d} t: u \in B\right\}\right) \leq \int_{J} \alpha(B(t)) \mathrm{d} t .
$$

Lemma 2.3 [16]. Let $B \subset C(J, E)$ be a bounded set on $J$. Then $\alpha(B(t)) \leq 2 \alpha(B)$.

Now we define an integral operator $S: C(J, E) \rightarrow C(J, E)$ by

$$
S v(t)=\int_{0}^{1} H(t, \tau) v(\tau) \mathrm{d} \tau
$$

Then, $S$ is linear continuous operator and by the expressed of $H(t, s)$, we have

$$
\left\{\begin{array}{l}
(S v)^{\prime \prime}(t)=-v(t), \quad 0<t<1 \\
\alpha_{1}(S v)(0)-\beta_{1}(S v)^{\prime}(0)=0 \\
\gamma_{1}(S v)(1)+\delta_{1}(S v)^{\prime}(1)=0
\end{array}\right.
$$

Lemma 2.4. The Sturm-Liouville BVP (1.1) has a positive solution if and only if the following integral-differential boundary value problem has a positive solution of 


$$
\left\{\begin{array}{l}
\frac{1}{p(t)}\left(p(t) v^{\prime}(t)\right)^{\prime}+f(S v(t))=0, \quad 0<t<1 \\
\alpha_{2} v(0)-\lim _{t \rightarrow 0+} \beta_{2} p(t) v^{\prime}(t)=0 \\
\gamma_{2} v(1)+\lim _{t \rightarrow 1-} \delta_{2} p(t) v^{\prime}(t)=0
\end{array}\right.
$$

where $S$ is given in (2.4).

Proof In fact, if $u$ is a positive solution of (1.1), let $u=S v$, then $v=-u^{\prime \prime}$. This implies $u^{\prime \prime}=-v$ is a solution of (2.6). Conversely, if $v$ is a positive solution of (2.6). Let $u=S v$, by (2.5), $u^{\prime \prime}=(S v)^{\prime \prime}=-v$. Thus, $u=S v$ is a positive solution of (1.1). This completes the proof of Lemma 2.1 .

So, we only need to concentrate our study on (2.6). Now, for the given $[a, b] \subset(0$, 1), $\rho$ as above in (II), we introduce

$$
K=\{u \in C(J, P): u(t) \geq \rho u(s), \quad t \in[a, b], \quad s \in[0,1]\} .
$$

It is easy to check that $K$ is a cone in $C[0,1]$. Further, for $u(t) \in K, t \in[a, b]$, we have by normality of cone $P$ with normal constant 1 that $\|u(t)\|_{1} \geq \rho\|u\|$.

Next, we define an operator $T$ given by

$$
T v(t)=\int_{0}^{1} G(t, s) p(s) f(S v(s)) \mathrm{d} s, \quad t \in[0,1]
$$

Clearly, $v$ is a solution of the BVP (2.6) if and only if $v$ is a fixed point of the operator $T$.

Through direct calculation, by (II) and for $v \in K, t \in[a, b], s \in J$, we have

$$
\begin{aligned}
T v(t) & =\int_{0}^{1} G(t, s) p(s) f(S v(s)) \mathrm{d} s \\
& \geq \rho \int_{0}^{1} G(s, s) p(s) f(S v(s)) \mathrm{d} s=\rho T v(s) .
\end{aligned}
$$

So, this implies that $T K \subset K$.

Lemma 2.5. Assume that $\left(H_{1}\right),\left(H_{2}\right)$ hold. Then $T: K \rightarrow K$ is strict set contraction.

Proof Firstly, The continuity of $T$ is easily obtained. In fact, if $v_{n}, v \in K$ and $v_{n} \rightarrow v$ in the sup norm, then for any $t \in J$, we get

$$
\left\|T v_{n}(t)-T v(t)\right\|_{1} \leq\left\|f\left(S v_{n}(t)\right)-f(S v(t))\right\|_{1} \int_{0}^{1} G(s, s) p(s) \mathrm{d} s,
$$

so, by the continuity of $f, S$, we have

$$
\left\|T v_{n}-T v\right\|=\sup _{t \in J}\left\|T v_{n}(t)-T v(t)\right\|_{1} \rightarrow 0 .
$$

This implies that $T v_{n} \rightarrow T v$ in the sup norm, i.e., $T$ is continuous.

Now, let $B \subset K$ is a bounded set. It follows from the the continuity of $S$ and $\left(\mathrm{H}_{2}\right)$ that there exists a positive number $L$ such that $\|f(S v(t))\|_{1} \leq L$ for any $v \in B$. Then, we can get 


$$
\|T v(t)\|_{1} \leq L \lambda<1, \quad \forall t \in J, \quad v \in B .
$$

So, $T(B) \subset K$ is a bounded set in $K$.

For any $\varepsilon>0$, by $\left(H_{1}\right)$, there exists a $\delta^{\prime}>0$ such that

$$
\int_{0}^{\delta^{\prime}} G(s, s) p(s) \leq \frac{\varepsilon}{6 L}, \quad \int_{1-\delta^{\prime}}^{1} G(s, s) p(s) \leq \frac{\varepsilon}{6 L} .
$$

Let $P=\max _{t \in\left[\delta^{\prime}, 1-\delta^{\prime}\right]} p(t)$. It follows from the continuity of $G(t, s)$ on $[0,1] \times[0,1]$ that there exists $\delta>0$ such that

$$
\left|G(t, s)-G\left(t^{\prime}, s\right)\right| \leq \frac{\varepsilon}{3 P L},\left|t-t^{\prime}\right|<\delta, \quad t, t^{\prime} \in[0,1]
$$

Consequently, when $\left|t-t^{\prime}\right|<\delta, t, t^{\prime} \in[0,1], v \in B$, we have

$$
\begin{aligned}
& \left\|T v(t)-T v\left(t^{\prime}\right)\right\|_{1}=\left\|\int_{0}^{1}\left(G(t, s)-G\left(t^{\prime}, s\right)\right) p(s) f(S v(s)) \mathrm{d} s\right\|_{1} \\
& \leq \int_{0}^{\delta^{\prime}}\left|G(t, s)-G\left(t^{\prime}, s\right)\right| p(s)\|f(S v(s))\|_{1} \mathrm{~d} s \\
& +\int_{\delta^{1}}^{1-\delta^{\prime}}\left|G(t, s)-G\left(t^{\prime}, s\right)\right| p(s) \| f\left(S v(s) \|_{1} \mathrm{~d} s\right. \\
& \quad+\int_{1-\delta^{\prime}}^{1}\left|G(t, s)-G\left(t^{\prime}, s\right)\right| p(s)\|f(S v(s))\|_{1} \mathrm{~d} s \\
& \leq 2 L \int_{0}^{\delta^{\prime}} G(s, s) p(s) \mathrm{d} s+2 L \int_{1-\delta^{\prime}}^{1} G(s, s) p(s) \mathrm{d} s \\
& \quad+P L \int_{0}^{1}\left|G(t, s)-G\left(t^{\prime}, s\right)\right| \mathrm{d} s \\
& \leq \varepsilon .
\end{aligned}
$$

This implies that $T(B)$ is equicontinuous set on $J$. Therefore, by Lemma 2.1, we have

$$
\alpha(T(B))=\sup _{t \in J} \alpha(T(B)(t)) .
$$

Without loss of generality, by condition $\left(H_{1}\right)$, we may assume that $p(t)$ is singular at $t$ $=0,1$. So, There exists $\left\{a_{n_{i}}\right\},\left\{b_{n_{i}}\right\} \subset(0,1),\left\{n_{i}\right\} \subset N$ with $\left\{n_{i}\right\}$ is a strict increasing sequence and $\lim _{i \rightarrow+1} n_{i}=+1$ such that

$$
\begin{gathered}
0<\cdots<a_{n_{i}}<\cdots<a_{n_{1}}<b_{n_{1}}<\cdots<b_{n_{i}}<\cdots<1 ; \\
p(t) \geq n_{i}, \quad t \in\left(0, a_{n_{i}}\right] \cup\left[b_{n_{i}}, 1\right), \quad p\left(a_{n_{i}}\right)=p\left(b_{n_{i}}\right)=n_{i} ; \\
\lim _{i \rightarrow+1} a_{n_{i}}=0, \lim _{i \rightarrow+1} b_{n_{i}}=1 .
\end{gathered}
$$


Next, we let

$$
p_{n_{i}}(t)=\left\{\begin{array}{l}
n_{i}, \quad t \in\left(0, a_{n_{i}}\right] \cup\left[b_{n_{i}}, 1\right) \\
p(t), t \in\left[a_{n_{i}}, b_{n_{i}}\right]
\end{array}\right.
$$

Then, from the above discussion we know that $(p)_{n_{i}}$ is continuous on $J$ for every $i \in$ $N$ and

$$
p_{n_{i}}(t) \leq p(t) ; p_{n_{i}}(t) \rightarrow p(t), \quad \forall t \in(0,1) \quad \text { as } i \rightarrow+1 .
$$

For any $\varepsilon>0$, by (2.9) and $\left(H_{1}\right)$, there exists a $i_{0}$ such that for any $i>i_{0}$, we have that

$$
2 L \int_{0}^{a_{n_{i}}} G(s, s) p(s) \mathrm{d} s<\frac{\varepsilon}{2}, 2 L \int_{b_{n_{i}}}^{1} G(s, s) p(s) \mathrm{d} s<\frac{\varepsilon}{2} .
$$

Therefore, for any bounded set $B \subset C[J, E]$, by (2.4), we have $S(B) \subset B$. In fact, if $v \in$ $B$, there exists $D>0$ such that $\|v\| \leq D, t \in J$. Then by the properties of $H(t, s)$, we can have

$$
\|S v(t)\|_{1} \leq \int_{0}^{1} H(t, \tau)\|v(\tau)\|_{1} d \tau \leq D \int_{0}^{1} H(t, \tau) d \tau \leq D,
$$

i.e., $S(B) \subset B$.

Then, by Lemmas 2.2 and 2.3, $\left(H_{2}\right)$, the above discussion and note that $p_{n_{i}}(t) \leq p(t)$, $t \in(0,1)$, as $t \in J, i>i_{0}$, we know that

$$
\begin{aligned}
\alpha(T(B)(t))= & \alpha\left(\left\{\int_{0}^{1} G(t, s) p(s) f(S v(s)) \mathrm{d} s \in B\right\}\right) \\
\leq & \left.\alpha\left(\int_{0}^{1} G(t, s)\left[p(s)-p_{n_{i}}(s)\right] f(S v(s)) \mathrm{d} s \in B\right\}\right) \\
& \left.+\alpha\left(\int_{0}^{1} G(t, s) p_{n_{i}}(s) f(S v(s)) \mathrm{d} s \in B\right\}\right) \\
\leq & 2 L \int_{0}^{a_{n_{i}}} G(s, s) p(s) \mathrm{d} s+2 L \int_{b_{n_{i}}} G(s, s) p(s) \mathrm{d} s \\
& +\int_{0}^{1} \alpha\left(G(t, s) p_{n_{i}}(s) f(S v(s)) \in B\right) \mathrm{d} s \\
\leq & \varepsilon+\int_{0}^{1} G(s, s) p(s) \alpha(f(S v(s)) \in B) \mathrm{d} s \\
\leq & \varepsilon+2 M \lambda \alpha(B) .
\end{aligned}
$$

Since the randomness of $\varepsilon$, we get

$$
\alpha(T(B)(t)) \leq 2 M \lambda \alpha(B), \quad t \in J
$$


So, it follows from (2.8) (2.11) that for any bounded set $B \subset C[J, E]$, we have

$$
\alpha(T(B)) \leq 2 M \lambda \alpha(B) .
$$

And note that $2 M \lambda<1$, we have $T: K \rightarrow K$ is a strict set contraction. The proof is completed.

Remark 1. When $E=\mathbf{R},(2.3)$ naturally hold. In this case, we may take $M$ as 0 , consequently,

$T: K \rightarrow K$ is a completely continuous operator. So, our condition $\left(H_{1}\right)$ is weaker than those of the above mention articles.

Our main tool of this article is the following fixed point theorem of cone.

Theorem 2.1 [16]. Suppose that $E$ is a Banach space, $K \subset E$ is a cone, let $\Omega_{1}, \Omega_{2}$ be two bounded open sets of $E$ such that $\theta \in \Omega_{1}, \bar{\Omega}_{1} \subset \Omega_{2}$. Let operator $T: K \cap\left(\bar{\Omega}_{2} \backslash \Omega_{1}\right) \rightarrow K$ be strict set contraction. Suppose that one of the following two conditions hold,

(i) $\|T x\| \leq\|x\|, \forall x \in K \cap \partial \Omega_{1},\|T x\| \geq\|x\|, \forall x \in K \cap \partial \Omega_{2}$;

(ii) $\|T x\| \geq\|x\|, \forall x \in K \cap \partial \Omega_{1},\|T x\| \leq\|x\|, \forall x \in K \cap \partial \Omega_{2}$.

Then, $T$ has at least one fixed point in $K \cap\left(\bar{\Omega}_{2} \backslash \Omega_{1}\right)$.

Theorem 2.2 [16]. Suppose $E$ is a real Banach space, $K \subset E$ is a cone, let $\Omega_{r}=\{u \in$ $K:\|u\| \leq r\}$. Let operator $T: \Omega_{r} \rightarrow K$ be completely continuous and satisfy $T x \neq x, \forall$ $x \in \partial \Omega_{r}$. Then

(i) If $\|T x\| \leq\|x\|, \forall x \in \partial \Omega_{r}$, then $i\left(T, \Omega_{r}, K\right)=1$;

(ii) If $\|T x\| \geq\|\mathrm{x}\|, \forall x \in \partial \Omega_{r}$, then $i\left(\mathrm{~T}, \Omega_{r}, K\right)=0$.

\section{The main results}

Denote

$$
f_{0}=\lim _{\|x\|_{1} \rightarrow 0+} \frac{\|f(x)\|_{1}}{\|x\|_{1}}, f_{\infty}=\lim _{\|x\|_{1} \rightarrow \infty} \frac{\|f(x)\|_{1}}{\|x\|_{1}} .
$$

In this section, we will give our main results.

Theorem 3.1. Suppose that conditions $\left(H_{1}\right),\left(H_{2}\right)$ hold. Assume that $f$ also satisfy

$$
\left(A_{1}\right): f(x) \geq r u^{*}, \xi\left\|\int_{0}^{1} H(\tau, \tau) x(\tau) \mathrm{d} \tau\right\|_{1} \leq\|x\|_{1} \leq r ;
$$

$\left(A_{2}\right): f(x) \leq R u^{*}, 0 \leq\|x\|_{1} \leq R$

where $u^{*}$ and $u^{*}$ satisfy

$$
\rho\left\|\int_{a}^{b} G(s, s) p(s) u^{*}(s) \mathrm{d} s\right\|_{1} \geq 1,\left\|u_{*}(s)\right\|_{1} \int_{0}^{1} G(s, s) p(s) \mathrm{d} s \leq 1 .
$$

Then, the boundary value problem (1.1) has a positive solution.

Proof of Theorem 3.1. Without loss of generality, we suppose that $r<R$. For any $u$ $\in K$, we have

$$
\|u(t)\|_{1} \geq \rho\|u\|, \quad t \in[a, b] .
$$

we define two open subsets $\Omega_{1}$ and $\Omega_{2}$ of $E$

$$
\Omega_{1}=\{u \in K:\|u\|<r\}, \Omega_{2}=\{u \in K:\|u\|<R\}
$$


For $u \in \partial \Omega_{1}$, by (3.1), we have

$$
r=\|u\| \geq\|u(s)\|_{1} \geq \rho\|u\|=\rho r, \quad s \in[a, b] .
$$

Then, for $u \in K \cap \partial \Omega_{1}$, by (2.4), (3.2), (II), for any $s \in[a, b], u \in K \cap \partial \Omega_{1}$, we have

$$
\begin{aligned}
r & \geq \int_{0}^{1} H(\tau, \tau)\|u(\tau)\|_{1} \mathrm{~d} \tau \geq\left\|\int_{0}^{1} H(\tau, \tau) u(\tau) \mathrm{d} \tau\right\|_{1} \geq\|S u(s)\|_{1} \\
& =\left\|\int_{0}^{1} H(s, \tau) u(\tau) \mathrm{d} \tau\right\|_{1} \geq \xi\left\|\int_{0}^{1} H(\tau, \tau) u(\tau) \mathrm{d} \tau\right\|_{1} .
\end{aligned}
$$

So, for $u \in K \cap \partial \Omega_{1}$, if $\left(\mathrm{A}_{1}\right)$ holds, we have

$$
\|\mathrm{Tu}(t)\|_{1}=\left\|\int_{0}^{1} G(t, s) p(s) f(S u(s)) \mathrm{d} s\right\|_{1} \geq r \rho\left\|\int_{a}^{b} G(s, s) p(s) u^{*}(s) r \mathrm{~d} s\right\|_{1} \geq r=\|u\| .
$$

Therefore, we have

$$
\|T u\| \geq\|u\|, \quad \forall u \in \partial \Omega_{1} .
$$

On the other hand, as $u \in K \cap \partial \Omega_{2}$, by (2.4), (3.2), (II), for any $s \in[a, b], u \in K \cap$ $\partial \Omega_{2}$, we have

$$
R \geq \int_{0}^{1} H(\tau, \tau)\|u(\tau)\|_{1} \mathrm{~d} \tau \geq\left\|\int_{0}^{1} H(\tau, \tau) u(\tau) \mathrm{d} \tau\right\|_{1} \geq\|S u(s)\| \geq 0 .
$$

For $u \in K \cap \partial \Omega_{2}$, if $\left(\mathrm{A}_{2}\right)$ holds, we know

$$
\begin{aligned}
\|T u(t)\|_{1} & =\left\|\int_{0}^{1} G(t, s) p(s) f(S u(s)) \mathrm{d} s\right\|_{1} \leq\left\|\int_{0}^{1} G(t, s) p(s) u_{*}(s) \mathrm{d} s\right\|_{1} R \\
& \leq \int_{0}^{1} G(t, s) p(s)\left\|u_{*}(s)\right\|_{1} \mathrm{~d} s R \leq \int_{0}^{1} G(s, s) p(s) \mathrm{d} s\left\|u_{*}(s)\right\|_{1} R \leq R=\|u\| .
\end{aligned}
$$

Thus

$$
\|T(u)\| \leq\|u\|, \quad \forall u \in \partial \Omega_{2} .
$$

Therefore, by (3.2), (3.3), Lemma 2.5 and $r<R$, we have that $T$ has a fixed point $v \in\left(\Omega_{2} \backslash \bar{\Omega}_{1}\right)$. Obviously, $v$ is positive solution of problem (2.6).

Now, by Lemma 2.4 we see that $u=S v$ is a position solution of BVP (1.1). The proof of Theorem 3.1 is complete.

Theorem 3.2. Suppose that conditions $\left(H_{1}\right),\left(H_{2}\right)$ and $\left(A_{1}\right)$ in Theorem 3.1 hold. Assume that $f$ also satisfy

$\left(A_{3}\right): f_{0}=0$;

$\left(A_{4}\right): f_{\infty}=0$.

Then, the boundary value problem (1.1) have at least two solutions. 
Proof of Theorem 3.2. First, by condition $\left(A_{3}\right),(2.4)$ and the property of limits, we can have

$\lim _{\|u\|_{1} \rightarrow 0+} \mid f(S u)\left\|_{1} /\right\| u \|_{1}=0$. Then, for any $m>0$ such that $m \int_{a}^{b} G(s, s) p(s) \mathrm{d} s \leq 1$, there exists a constant $\rho_{*} \in(0, r)$ such that

$$
\|f(S u)\|_{1} \leq m\|u\|_{1}, \quad 0<\|u\|_{1} \leq \rho_{*}, \quad u \neq 0 .
$$

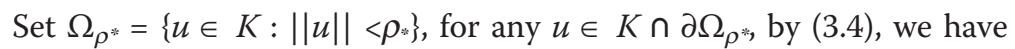

$$
\|f(S u)\|_{1} \leq m\|u\|_{1} \leq m \rho_{*}
$$

For $u \in K \cap \partial \Omega \rho^{*}$, we have

$$
\begin{aligned}
\|T u(t)\|_{1} & =\left\|\int_{0}^{1} G(t, s) p(s) f(S u(s)) \mathrm{d} s\right\|_{1} \leq \int_{0}^{1} G(t, s) p(s)\|f(S u(s))\|_{1} \mathrm{~d} s \\
& \leq \int_{a}^{b} G(t, s) p(s) m \rho_{*} \mathrm{~d} s \leq \rho_{*} m \int_{a}^{b} G(s, s) p(s) \mathrm{d} s \leq \rho_{*}=\|u\| .
\end{aligned}
$$

Therefore, we can have

$$
\|T u\| \leq\|u\|, \quad \forall u \in \partial \Omega_{\rho_{*}} .
$$

Then by Theorem 2.2, we have

$$
i\left(T, \Omega_{\rho_{*}}, K\right)=1 \text {. }
$$

Next, by condition $\left(A_{4}\right),(2.4)$ and the property of limits, we can have $\lim _{\|u\|_{1} \rightarrow 1}\|f(S u)\|_{1} /\|u\|_{1}=0$. Then, for any $\bar{m}>0$ such that $\bar{m} \int_{a}^{b} G(s, s) p(s) \mathrm{d} s \leq 1$, there exists a constant $\rho_{0}>0$ such that

$$
\|f(S u)\|_{1} \leq \bar{m}\|u\|_{1}, \quad\|u\|_{1}>\rho_{0} .
$$

We choose a constant $\rho^{*}>\max \left\{r, \rho_{0}\right\}$, obviously, $\rho_{*}<\mathrm{r}<\rho^{*}$. Set $\Omega_{\rho *}=\left\{u \in K:\|u\|<\rho^{*}\right\}$, for any $u \in K \cap \partial \Omega_{\rho^{*}}$, by (3.6), we have

$$
\|f(S u)\|_{1} \leq \bar{m}\|u\|_{1} \leq \bar{m} \rho^{*} .
$$

For $u \in K \cap \partial \Omega \rho^{*}$, we have

$$
\begin{aligned}
\|T u(t)\|_{1} & =\left\|\int_{0}^{1} G(t, s) p(s) g(s) f(S u(s)) \mathrm{d} s\right\|_{1} \leq \int_{0}^{1} G(t, s) p(s) g(s)\|f(S u(s))\|_{1} \mathrm{~d} s \\
& \leq \int_{a}^{b} G(t, s) p(s) \bar{m} \rho^{*} \mathrm{~d} s \leq \rho^{*} \bar{m} \int_{a}^{b} G(s, s) p(s) \mathrm{d} s \leq \rho^{*}=\|u\| .
\end{aligned}
$$

Therefore, we can have

$$
\|T u\| \leq\|u\|, \quad \forall u \in \partial \Omega_{\rho *} .
$$

Then by Theorem 2.2, we have

$$
i\left(T, \Omega_{\rho *}, K\right)=1 .
$$


Finally, set $\Omega_{r}=\{u \in K:\|u\|<\mathrm{r}\}$, For any $u \in \partial \Omega_{r}$, by $\left(A_{2}\right)$, Lemma 2.2 and also similar to the latter proof of Theorem 3.1, we can also have

$$
\|T u\| \geq\|u\|, \quad \forall u \in \partial \Omega_{r} .
$$

Then by Theorem 2.2, we have

$$
i\left(T, \Omega_{r}, K\right)=0 .
$$

Therefore, by (3.5), (3.7), (3.8), and $\rho_{*}<r<\rho^{*}$, we have

$$
i\left(T, \Omega_{r} \backslash \bar{\Omega}_{\rho_{*}}, k\right)=-1, i\left(T, \Omega_{\rho *} \backslash \bar{\Omega}_{r}, k\right)=1 .
$$

Then $T$ have fixed point $v_{1} \in \Omega_{r} \backslash \bar{\Omega}_{\rho *}$, and fixed point $v_{2} \in \Omega_{\rho *} \backslash \bar{\Omega}_{r}$. Obviously, $v_{1}$, $v_{2}$ are all positive solutions of problem (2.6).

Now, by Lemma 2.4 we see that $u_{1}=S v_{1}, u_{2}=S v_{2}$ are two position solutions of BVP (1.1). The proof of Theorem 3.2 is complete.

\section{Application}

In this section, in order to illustrate our results, we consider some examples.

Now, we consider the following concrete second-order singular BVP (SBVP)

Example 4.1. Consider the following SBVP

$$
\left\{\begin{array}{l}
\frac{3}{\sqrt[3]{t}}\left(\frac{1}{3} \sqrt[3]{t} u^{\prime \prime \prime}(t)\right)^{\prime}+160\left[u^{\frac{1}{2}}+u^{\frac{1}{3}}\right]=\theta, \quad 0<t<1, \\
u(0)-3 u^{\prime}(0)=\theta, \quad u(1)+2 u^{\prime}(1)=\theta, \\
3 u^{\prime \prime}(0)-\lim _{t \rightarrow 0^{+}} \frac{1}{3} \sqrt[3]{t} u^{\prime \prime \prime}(t)=\theta, \quad u^{\prime \prime}(1)+\lim _{t \rightarrow 1^{-}} \frac{1}{3} \sqrt[3]{t} u^{\prime \prime \prime}(t)=\theta,
\end{array}\right.
$$

where

$$
\begin{gathered}
\alpha_{1}=\gamma_{1}=1, \beta_{1}=3, \delta_{1}=2, \beta_{2}=\gamma_{2}=\delta_{2}=1, \alpha_{2}=3, \\
p(t)=\frac{1}{3} \sqrt[3]{t}, f(u)=160\left(u^{\frac{1}{2}}+u^{\frac{1}{3}}\right) .
\end{gathered}
$$

Then obviously,

$$
\int_{0}^{1} \frac{1}{p(t)} \mathrm{d} t=\frac{3}{2}, f_{\infty}=0, \quad f_{0}=0
$$

By computing, we know that the Green's function are

$$
\begin{aligned}
& H(t, s)=\frac{1}{6}\left\{\begin{array}{l}
(3+s)(3-t), 0 \leq s \leq t \leq 1 \\
(3+t)(3-s), 0 \leq t \leq s \leq 1
\end{array}\right. \\
& G(t, s)=\frac{1}{7}\left\{\begin{array}{l}
(1+3 s)(2-t), 0 \leq s \leq t \leq 1 \\
(1+3 t)(2-s), 0 \leq t \leq s \leq 1 .
\end{array}\right.
\end{aligned}
$$

It is easy to note that $0 \leq G(s, s) \leq 1$ and conditions $\left(H_{1}\right),\left(H_{2}\right),\left(A_{3}\right),\left(A_{4}\right)$ hold.

Next, by computing, we know that $\rho=0.44, \xi=0.8$. We choose $r=3, u^{*}=104$, as $1.05=\rho \xi r \leq\|u\|=\max \{u(t), t \in J\} \leq 3$ and $\rho\left\|\int_{a}^{b} G(s, s) p(s) u^{*}(s) \mathrm{d} s\right\|=1.3>1$, because of the monotone increasing of $f(u)$ on $[0, \infty)$, then 


$$
f(u) \geq f(1.05)=326.4, \quad 1.05 \leq\|u\| \leq 3 .
$$

Therefore, as

$$
\rho \xi r=\rho \xi\left\|\int_{0}^{1} H(\tau, \tau)\right\| u\|\mathrm{~d} \tau\| \leq \xi\left\|\int_{0}^{1} H(\tau, \tau) u(\tau) \mathrm{d} \tau\right\|,
$$

so we have

$$
f(u) \geq r u^{*}, \xi\left\|\int_{0}^{1} H(\tau, \tau) u(\tau) \mathrm{d} \tau\right\| \leq\|u\| \leq r
$$

then conditions $\left(A_{1}\right)$ holds. Then by Theorem 3.2, SBVP (4.1) has at least two positive solutions $u_{1}, u_{2}$ and $0<\left\|u_{1}\right\|<3<\left\|u_{2}\right\|$.

\section{Acknowledgements}

The authors would like to thank the reviewers for their valuable comments and constructive suggestions. HS and LL were supported financially by the Shandong Province Natural Science Foundation (ZR2009AQ004), NSFC (11026108, 11071141) and XW was supported by the Shandong Province planning Foundation of Social Science (09BJGJ14), Shandong Province Natural Science Foundation (Z2007A04).

\section{Author details}

${ }^{1}$ School of Mathematic and Quantitative Economics, Shandong University of Finance and Economics, Jinan Shandong 250014, China ${ }^{2}$ School of Mathematical Sciences, Qufu Normal University, Qufu Shandong 273165, China ${ }^{3}$ School of Economics, Shandong University, Jinan Shandong 250014, China

\section{Authors' contributions}

All authors contributed equally to the manuscript and read and approved the final manuscript.

\section{Competing interests}

We declare that we have no significant competing financial, professional, or personal interests that might have influenced the performance or presentation of the work described in this manuscript.

\section{Received: 12 October 2011 Accepted: 21 December 2011 Published: 21 December 2011}

\section{References}

1. Ma, RY, Wang, HY: On the existence of positive solutions of fourth order ordinary differential equation. Appl Anal. 59, 225-231 (1995). doi:10.1080/00036819508840401

2. Schroder, J: Fourth-order two-point boundary value problems: estimates by two side bounds. Nonlinear Anal. 8 107-144 (1984). doi:10.1016/0362-546X(84)90063-4

3. Agarwal, RP, Chow, MY: Iterative methods for a fourth order boundary value problem. J Comput Appl Math. 10, 203-217 (1984). doi:10.1016/0377-0427(84)90058-X

4. Agarwal, RP: On the fourth-order boundary value problems arising in beam analysis. Diff Integral Equ. 2, 91-110 (1989)

5. Ma, RY: Positive solutions of fourth-order two point boundary value problem. Ann Diff Equ. 15, 305-313 (1999)

6. O'Regan, D: Solvability of some fourth (and higher) order singular boundary value problems. J Math Anal Appl. 161, 78-116 (1991). doi:10.1016/0022-247X(91)90363-5

7. Gupta, CP: Existence and uniqueness theorems for a bending of an elastic beam equation at resonance. J Math Anal Appl. 135, 208-225 (1988). doi:10.1016/0022-247X(88)90149-7

8. Gupta, CP: Existence and uniqueness theorems for a bending of an elastic beam equation. Appl Anal. 26, 289-304 (1988). doi:10.1080/00036818808839715

9. Yang, YS: Fourth order two-point boundary value problem. Proc Am Math Soc. 104, 175-180 (1988). doi:10.1090/S00029939-1988-0958062-3

10. Li, H, Sun, J: Positive solutions of sublinear Sturm-Liouville problems with changing sign nonlinearity. Comput Math Appl. 58, 1808-1815 (2009). doi:10.1016/j.camwa.2009.07.059

11. Yang, J, Wei, Z, Liu, K: Existence of symmetric positive solutions for a class of Sturm-Liouville-like boundary value problems. Appl Math Comput. 214, 424-432 (2009). doi:10.1016/j.amc.2009.04.008

12. $\mathrm{Xu}, \mathrm{F}, \mathrm{Su}, \mathrm{H}$ : Positive solutions of fourth-order nonlinear singular boundary value problems. Nonlinear Anal. 68(5), 1284-1297 (2008). doi:10.1016/j.na.2006.12.021

13. Liu, B: Positive solutions of fourth-order two point boundary value problem. Appl Math Comput. 148, 407-420 (2004). doi:10.1016/S0096-3003(02)00857-3

14. Wei, Z: Positive solutions of singular dirichlet boundary value problems. Chin Ann Math 20(A), 543-552 (2007). in Chinese

15. Wong, PJY, Agarwal, RP: Eigenvalue of lidstone boundary value problems. Appl Math Comput. 104, 15-31 (1999). doi:10.1016/S0096-3003(98)10045-0 
16. Guo, DJ, Lakshmikantham, V: Nonlinear Problems in Abstract Cone. Academic Press, Inc, New York (1988)

17. Zhang, X: Positive solutions for three-point semipositone boundary value problems with convex nonlinearity. J Appl Math Comput. 30, 349-367 (2009). doi:10.1007/s12190-008-0177-6

doi:10.1186/1687-1847-2011-65

Cite this article as: Su et al:: Sturm-Liouville BVP in Banach space. Advances in Difference Equations 2011 2011:65.

Submit your manuscript to a SpringerOpen ${ }^{\odot}$ journal and benefit from:

- Convenient online submission

$\rightarrow$ Rigorous peer review

- Immediate publication on acceptance

- Open access: articles freely available online

- High visibility within the field

- Retaining the copyright to your article

Submit your next manuscript at $\boldsymbol{\nabla}$ springeropen.com 